\title{
The Rolled-up and Tip Vortices Studies in the CFD Model of the 3-D Swept-Backward Wind Turbine Blades
}

\author{
Sutrisno ${ }^{1}$, Deendarlianto ${ }^{1}$, Tri Agung Rochmat ${ }^{1}$, Indarto ${ }^{1}$, Setyawan Bekti Wibowo ${ }^{1,2}$, Sigit Iswahyudi ${ }^{1,3}$, \\ Caesar Wiratama ${ }^{1} \&$ Djatmiko Bagus Maulana Erlambang ${ }^{1}$ \\ ${ }^{1}$ Department of Mechanical and Industrial Engineering, Faculty of Engineering, Universitas Gadjah Mada, \\ Yogyakarta 55281, Indonesia \\ ${ }^{2}$ Department of Mechanical Engineering, Vocation Program, Universitas Gadjah Mada, Yogyakarta 55281, \\ Indonesia \\ ${ }^{3}$ Department of Mechanical Engineering, Universitas Tidar, Magelang, Indonesia \\ Correspondence: Sutrisno, Department of Mechanical and Industrial Engineering, Faculty of Engineering \\ Universitas Gadjah Mada, Yogyakarta 55281, Indonesia. E-mail: mrsutrisnougm@gmail.com
}

Received: October 29, 2017

doi:10.5539/mas.v11n12p118

Accepted: November 14, 2017

Online Published: November 30, 2017

URL: https://doi.org/10.5539/mas.v11n12p118

The research is financed by The Department of Higher Education, The Republic of Indonesia.

\begin{abstract}
In this paper, the method to analyze of vortex dynamics simulation of 3-D (three dimensional) backward wind turbine blades is introduced, consisted of flow visualization part and detailed measurement part. With this method, one could explain visually and by calculation the role of 3-D flow vortex mechanism patterns on 3-D backward wind turbine blade, the interchange between kinetic and potential energies, the utilization of very strong vortex, which could lose energy, generate lift, and produce tangential mechanical power. The method could be elucidated by analyzing the appearance of rolled-up vortex effect on the 3-D backward wind turbine blades. A sharp pointed backward blade, generally has a weak tip vortex, may generate a second weak vortex center, and appears due to the rolled-up vortex effect, which is quite difficult to identify. The weakness of tip vortex makes the sharp pointed blade more efficient to exchange energy. Blunt backward turbine blades generally have a strong vortex center, a tip vortex; which in the form of a vortex core. Due to the rolled-up vortex effect, it could generate a second weak vortex center that is clearly visible.
\end{abstract}

Keywords: CFD model, Q-criterion, backward, 3-D wind turbine blades, rolled-up vortex, vortex core, accelerated region, vortex dynamics simulation analyze method

\section{Introduction}

\subsection{From Stall-Delay to Rolled-Up Vortex and Vortex Core}

The research on three dimensional (3-D) wind turbine blades has been introduced. Sutrisno et al.(Sutrisno, Prajitno, Purnomo, \& B.W. Setyawan, 2016) has initiated a research on the performance and the flow visualization of 3-D wind turbine blade models by fastening the backward and forward blade parts to the base and the tip of the wind turbine blades, so that the blades could affect the growths of the blade stall delay when it occurs. Furthermore, in their following report (Sutrisno et al., 2017), it is realized that stall delays, identified in 3-D wind turbine forward and backward blade models, are due to the effects of the rolled-up vortex generated by delta formed-hubs. The conclusion has been drawn based on several experimental evidences (Gursul, Wang, \& Vardaki, 2007)(Nelson \& Pelletier, 2003)(Han \& Kinnas, 2013).

Sutrisno et al. (Sutrisno, Rochmat, et al., n.d.) have realized how the strength of vortex core produced by rolled-up vortices should be intensively investigated especially its effect on the farther regions affected. Further, in order to carry out three-dimensional CFD measurements, one could employ Q-criterion (Calderon, D.E, Wang, Z, \& Gursul, 2012). As Chen presented of the effect of sideslip in a fighter model, the strength of rolled-up vortex effects came into view. The resultant improvement of the pressure field on the wing (Mitchell \& Delery, 2001) or on other surface yields lift enhancement which needs practical verification. 
The main principles of the vortex dynamics mechanism in aerodynamic areas have been deeply applied in helicopter blade designs and in the interaction of helical tip and root vortices in wind turbines. A review of helicopter rotor blade tip shape studies has been conducted (Brocklehurst \& Barakos, 2013). Some investigations on the root vortices in a wind turbine wake have been also studied. The design and dynamic of helicopter rotor blades have been thoroughly optimized. The interaction of helical tip and root vortices in a wind turbine wake have been learned (Sherry, Nemes, Lo Jacono, Blackburn, \& Sheridan, 2013). Characterization of a horizontal axis wind turbines tip and root vortices have been examined (Sherry, Sheridan, \& Jacono, 2013). Testing basic performance of a very small wind turbine designed for multi-purposes have been done (Hirahara, Hossain, \& Kawahashi, 2005). It is unfortunate that the significance of the rolled-up vortex effect, that is the quantified measurement of the vortex developed, the tip vortex and the vortex breakdown have not been evaluated, that one could not characterize more detailed about the strength of their vortices axial speed, negative pressure and trajectory of the vortex centers including their surface pressure distribution to generate effective tangential powers.

The rolled-up vortex phenomenon has been thoroughly investigated in aerospace and aeronautical sciences more intensively than in wind turbine blades. A wing-body configuration to delay the onset of vortex asymmetry designed has been explored (Cai, Tsai, Luo, \& Liu, 2011). Double delta fighter has been examined (Sun, Li, \& Zhang, 2013). Flow structure over the yawed non-slender diamond wing has been analyzed (Sahin, Yayla, Canpolat, \& Akilli, 2012). An integrated to UCAV stability \& control estimation X-31 has been studied (Cummings \& Schutte, 2010). Validation of static and forced motion flow physics of a canard configured TransCruiser has been simulated (Ghoreyshi, Korkis-kanaan, Jirásek, Cummings, \& Lofthouse, 2016). Wingtip vortex control has been learned (T. Lee \& Su, 2012). Vortical flow control over delta wing for different sweep back angles has been deeply explored (Kwak, Nelson, Dame, \& Dame, 2010). Turbulent structures in the trailing vortex wake of a delta wing have been thoroughly considered (Miller \& Williamson, 1997).

Several vortex dynamics controls by second-order turbulence and vortex mechanism in aircraft aerodynamics research have been extensively conducted. Ghoreyshi et al. (Ghoreyshi, Cummings, Ronch, \& Badcock, 2013) have investigated transonic aerodynamic load modeling of X-31 aircraft pitching motions. In order to validate the results of CFD modeling, the experimental water tunnel results, where its accuracy have been tested with Erm's method (Erm \& Ol, 2012), have been employed. The topology of the main vortex system and canard-wing interactions in subsonic flow have been conducted (Samimi, Davari, \& Soltani, 2013), the vortical flow control over delta wings with different sweep back angles have been studied (Kwak et al., 2010), the validation of static and forced motion flow physics of a canard configured TransCruiser have been simulated (Ghoreyshi et al., 2016), and experimental investigations on leading-edge vortex structures for flow over non-slender delta wings have been carried out (Jin-Jun \& Wang, 2008). It is ill-fated that, unparalleled within aeronautical and aerospace engineering, turbulent structures, topology, leading-edge vortex structure and second-order turbulence in wind turbine blades sciences, especially for the 3-D wind turbine blades, other than straight blades, have not been investigated as much.

\subsection{Turbulence Models with Q-Criterion Algorithm to Visualize the Dynamics of Vortex}

Rolled-up vortices generate vortex center, and when the sweep angle is adequate, then the vortex center could be transformed to become vortex core. Q-criterion could be employed to visualize and enumerate the vortex centers and vortex cores (Calderon, D.E et al., 2012)(Chen, Liu, Guo, \& Qu, 2015). Q-criterion could also be implemented to measure the vortex phenomenon, the strength of their vortices axial speed, negative pressure and trajectory of the vortex centers including their surface pressure distribution to generate effective tangential powers, and then one could identify the location of vortex breakdown by measuring the axial velocity along the vortex core (Chen et al., 2015). It is inopportune that, the employment of the Q-criterion algorithm so far have not been combined with the footprint of the streamline for the vortex development, that complete picture of the vortex dynamics has not been completed.

Haller (Haller, 2005) has precisely defined the Q-criterion, as well as a vortex. Employing the definition, the aerodynamic characteristics of a wind turbine blades with a sinusoidal leading edge have been investigated (Zhang \& Wu, 2012), by using iso-surface of the Q-criterion. Further, a flow separation control has been studied with the implementation of tubercles on a marine propeller blade (Ibrahim \& New, 2015). Tubercles are essentially sinusoidal serrations that are present in the fins of humpback whales. In this case, to isolate the vortical structures near the propeller, the Q-criterion value is set. In order to understand the performance and shape characteristics of a helical Savonius wind turbine, the turbine at various helical angles has been investigated (J. Lee, Lee, \& Lim, 2017). The power coefficient (Cp) at different tip speed ratios (TSRs) and torque coefficient (CT) at different azimuths were observed under the conditions of a constant projection area 
and aspect ratio. The Q-criterion method was used, and in the case of the Savonius wind turbine, vortex formation was in the direction of rotation.

\subsection{Q-criterion Algorithm Needs Suitable Gridding and Accuracy}

In aerospace science, some fighters have been simulated to investigate deeply inside the vortex dynamics and its turbulence using CFD simulation turbulence modeling. The nonlinear phenomenon of the rolled-up vortex is controlled and affected by a second-order mechanism in the size of Kolmogorov microscale. Turbulence models, Q-criterion algorithm needs appropriate gridding and accuracy.

Some turbulence models are very well-known, namely i) DNS model is solved directly at Kolmogorov microscale without any modeling, which is costly, ii) LES model would be good for separation but perform poorly in boundary layers, and iii) RANS would so effective in boundary layers in contrary do poorly in the mainstream (Chen et al., 2015). Detached eddy simulation (DES), which is a special choice, could also be employed; it combines RANS inside the boundary layers and LES to catch the separation in the large-scale turbulence field in the mainstream.

Detached eddy simulation (DES) requires numerical modeling with very small gridding in the boundary layer close to the wall boundary, therefore needs structured gridding with logarithmic meshes. Some successful applications have been conducted. The DES investigation of turbulence at a circular pier with scour hole has been carried out (Kirkil, Constantinescu, \& Ettema, 2009). Im and Zha (Im \& Zha, 2011) have studied the delayed DES of a stall flow over NACA0012 airfoil using high order schemes. The DES of the flow behind an isolated propeller have been simulated (Muscari, Di Mascio, \& Verzicco, 2013). Jee and Shariff (Jee \& Shariff, 2014) have conducted a DES based on the v2-f model. A DES for turbulent flows in a pipe with a snowflake fractal orifice has been learned (Nicolleau et al., 2012). Spalart (Spalart, 2009) has explored DES of F-15 jet at a $65^{\circ}$ angle of attack. Sun et al. (Sun et al., 2013) have modeled the DES on massively separated flows over a 76/40 - double-delta wing. A comprehensive study of DES has been conducted (Travin, Shur, Strelets, \& Spalart, 1999)(Morton et al., 2004)(Mockett, 2008). Detached eddy simulation (DES) of the flow, especially behind an isolated propeller, has been analyzed (Muscari \& Mascio, 2013). DES based on the v2-f model has been conducted (Jee \& Shariff, 2014). Squires (Squires, 2004) has reported the current status and perspectives of DES A comparative study of RANS, detached eddy simulation (DES) and large eddy simulation (LES) of internal torque converters flows has been investigated (Liu, Liu, \& Ma, 2015). Larsson and Wang (Larsson \& Wang, 2014) have enlightened the prospect of using LES and DES in engineering design, and the research required to get there.

This paper shows the CFD simulation using detached-eddy simulation (DES) to simulate the characteristic of 3-D backward wind turbine blade model, the blunt and the sharp edge one. The results show the complete illustration of their vortex dynamic phenomena, display the footprint of the streamline for the vortex development, the results of $\mathrm{Q}$ criterion algorithm employment to show vortex center, vortex core and breakdown location with respect to free stream velocity. The purpose of the investigation is to measure the strength of their vortices axial speed and negative pressure of the vortex centers including their surface pressure distribution to generate effective tangential powers, the strength of the vortex center the length of the vortex core, the loss of energy with respect to the tangential local blade velocity. The streamline distribution and pressure coefficient distribution would be utilized.

Therefore one could deeply comprehend the difference between the blunt and the sharp edge wind turbine blades, step by step the function of 3-D flow vortex mechanism patterns, the interchange between kinetic and potential energies, the utilization of very strong vortex could generate lift, produce tangential mechanical power.

\section{Numerical Procedures}

\subsection{Numerical Preparation}

The performances of the 3-D backward wind turbine blades model with blunt and sharp edge blades were simulated using 3-D CFD model. Computational domain was created; wind turbine blade CAD model was divided into blocks and then chopped to become mesh.

The computational study begins by creating computational models using CAD Software. The computational model of the 3-D backward wind turbine blades with a radius of $1,549 \mathrm{~m}$ refers to the actual model in the experiment as shown in Figure 1. The model has been exported into a universal CAD format file called The Initial Graphics Exchange Specification (IGES) read on the processing of computing nets. The next process is to prepare a structured computing network. The net structure uses a hexahedral structured mesh with H-shaped type to facilitate convergence and good results in obtaining vortex visualizations that occur along the turbine blade 
surface. The step of making computational mesh begins by dividing the turbine blade parts into several blocks according to the geometric changes and the inner parts of the turbine blade as shown in Figure 2. To reduce the computational load, the model design is made by making the domain periodically $120^{\circ}$, rather than creates two third turbine blades unnecessarily.

The computational mesh is based on a layer near the wall using a non-dimensional wall distance with a value of $\mathrm{y}+=4$ in which the first mesh thickness on the wall was $0.0002 \mathrm{~m}$. This value refers to the mesh independency test of previous studies conducted by comparing the results of the use from $y+=1$ to $y+=6$ and the result shows that the use of the smallest cell value near the wall from the value $y+=1$ to $y+=4$ did not experience a significant difference of associated vortex patterns that occur and changed after the larger cell size above $y+=5$ so the use of this reference value can save the computational load while the accuracy of the results is still good. The number of cells used in this turbine model reaches 1.2 million cells as shown in Figure 2. The number of cells used has gone through a study of the convergence of torque values. The study was conducted for the number of cells 800000 to 1.5 million and the result is convergent torque value since cell number above 1 million.

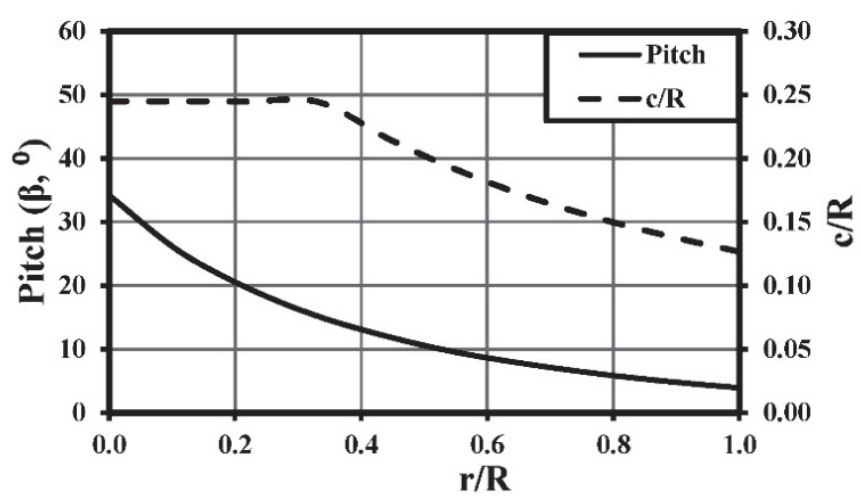

(a)

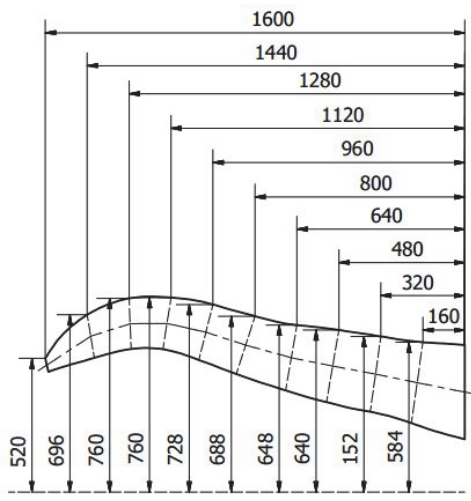

(b)

Figure 1. A 3-D backward wind turbine blade model with sharp edge blade used in this study, with $1.549 \mathrm{~m}$ dimensions refer to the actual size. (a) Pitch and chord distributions, (b) A view of the sharp edge blade model

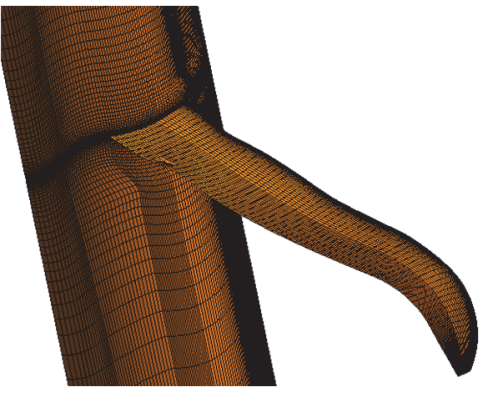

(a)

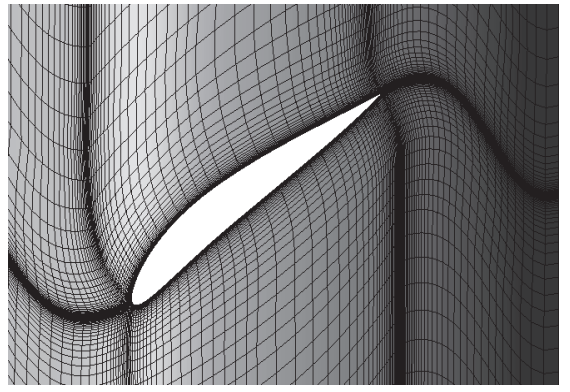

(b)

Figure 2. Blocking strategy in 3-D backward wind turbine blade model with sharp edge blade with H-shape mesh at blade surface 


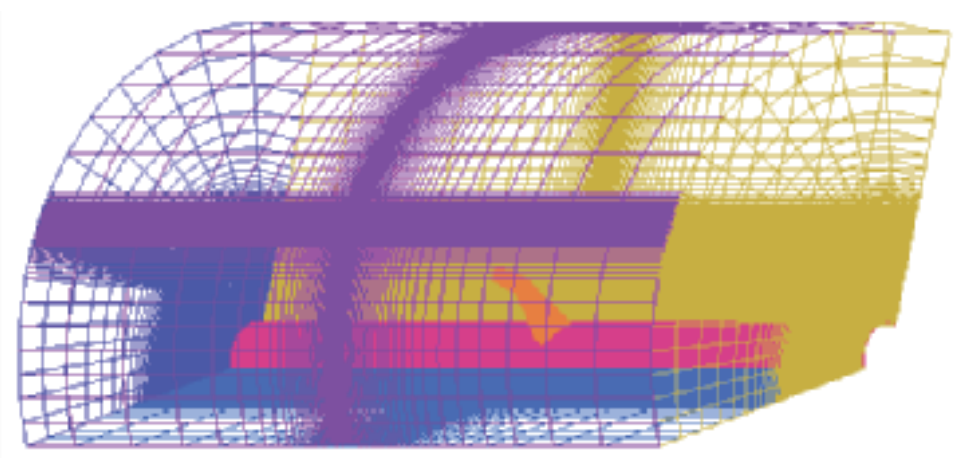

Figure 3. Blocking strategy in 3-D backward wind turbine blade model with sharp edge blade at computational domain with $120^{\circ}$ periodic boundary conditions

Computational domains are made in several boundary conditions such as inlet, outlet, hub, periodic, shroud, and blade as shown in Figure 3. In this case, the fluid condition is considered incompressible and steady state by neglecting the effect of gravity. To solve the computational model, the Navier-Stokes equations are used throughout the computational domain and use the "Moving Reference Frame" mode on the turbine wall.

Completed model computation and discretization model employ Semi Implicit-Method for Pressure Linked Equation method, while for turbulence model, the DES equation was used.

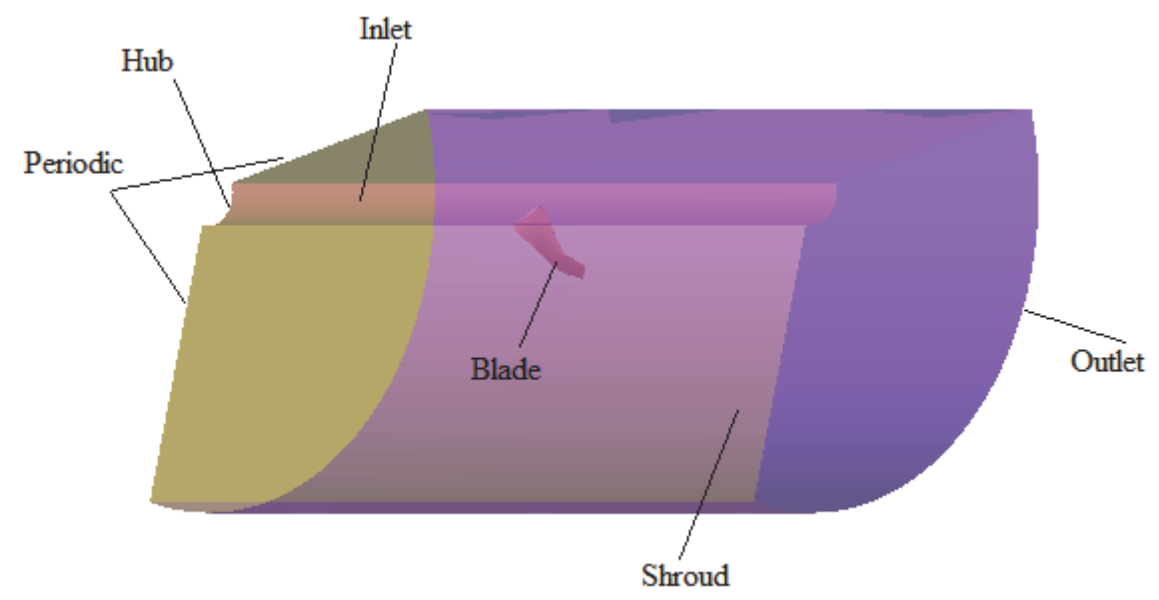

Figure 4 . The name of boundary condition at the computational domain

\section{Numerical Results}

This paper describes the aerodynamic characteristics of 3-dimensional blunt and sharp-edged, backward, wind turbine blades using CFD model, simulated using Fluent ANSYS in order to show flow feature of the tip vortex and rolled-up vortices structure. Q-criterion is used to capture the detailed of the vortex core phenomenon of the vortex center obtained by measuring the axial velocity along the vortex center. Graphs of axial velocity ratio $\mathrm{Uo} /$ Ulocal ( $\mathrm{z}$ ) and pressure coefficient of the cortex center can be analyzed to prove whether there is a vortex core in that area. 

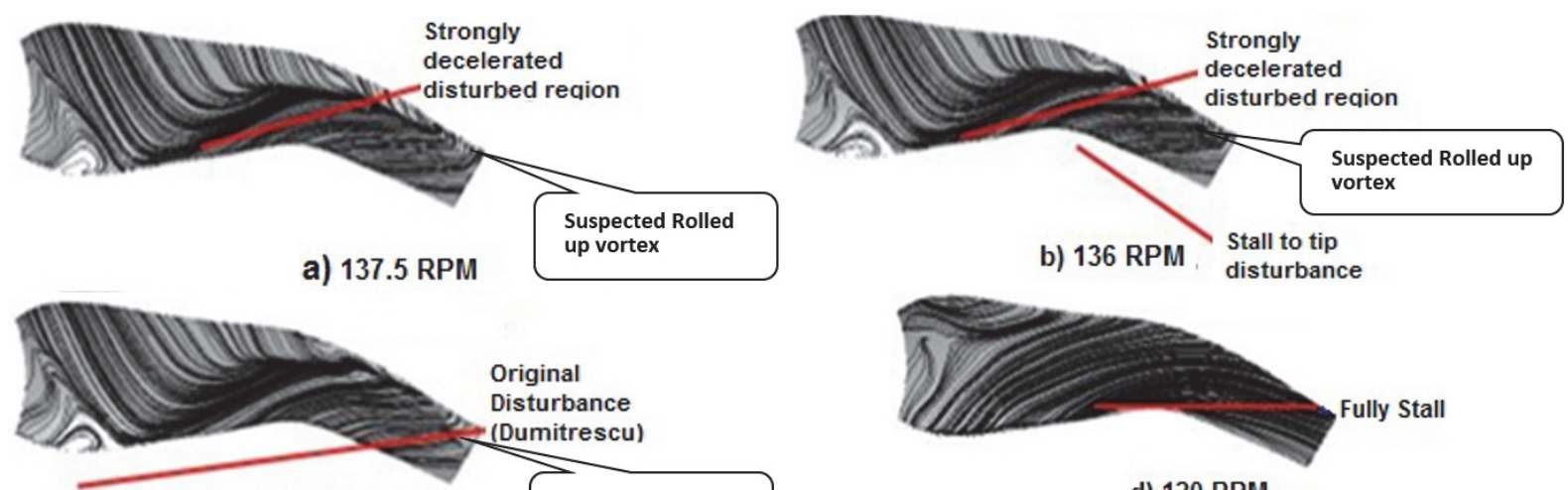

c) 135 RPM

\section{Original}

Disturbance

(Dumitrescu)
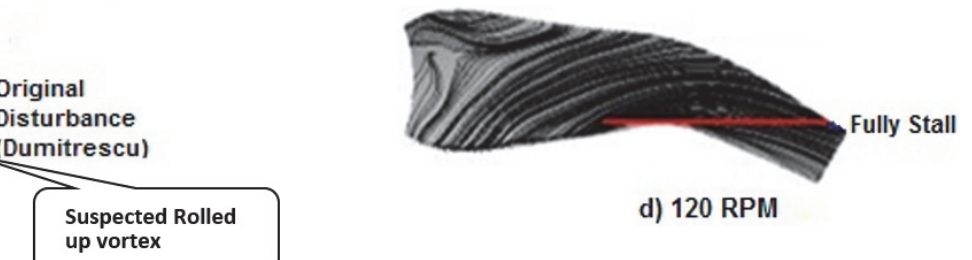

d) 120 RPM up vortex

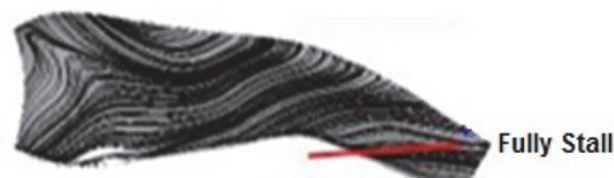

e) 90 RPM

Figure 5. Limiting Streamlines at the surface of the blade. The sample data was taken at a speed of $12 \mathrm{~m} / \mathrm{s}$ on the transition area with variation 137.5, 136, 135 RPM and 120 RPM. (Sutrisno, Deendarlianto, et al., no date)

\subsection{The Blunt Blade}

\subsubsection{Limiting Streamlines at the Surface of the Blunt Blade}

Fig. 5 illustrates the limiting streamline on the blade surface at a wind speed of $12 \mathrm{~m} / \mathrm{s}$ for some variations of rotation, among which 137.5, 136, 135 and 120 RPM (Sutrisno, Deendarlianto, et al., n.d.).In this figure, there is a noticeable change in the limiting streamline. At 137.5 RPM, the laminar plane is in the middle to the end of the blade surface, although increasingly narrowed further, this is because the bottom is the decelerated region while the upper part of the backward blade is an accelerated region. At a heavier load at 136 RPM, the rotation decreases and the more decelerated, therefore the laminar region further shifts downward as shown in Fig. 5b- c.

3.1.2 The visualization of the main \& minor vortex center 


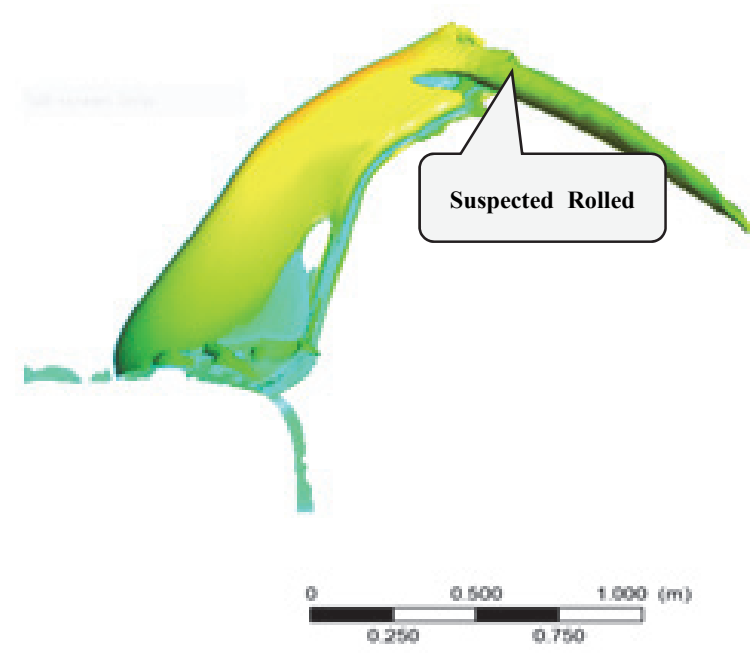

(a) Vortex core visualization at $12 \mathrm{~m} / \mathrm{s}, 140 \mathrm{RPM}$

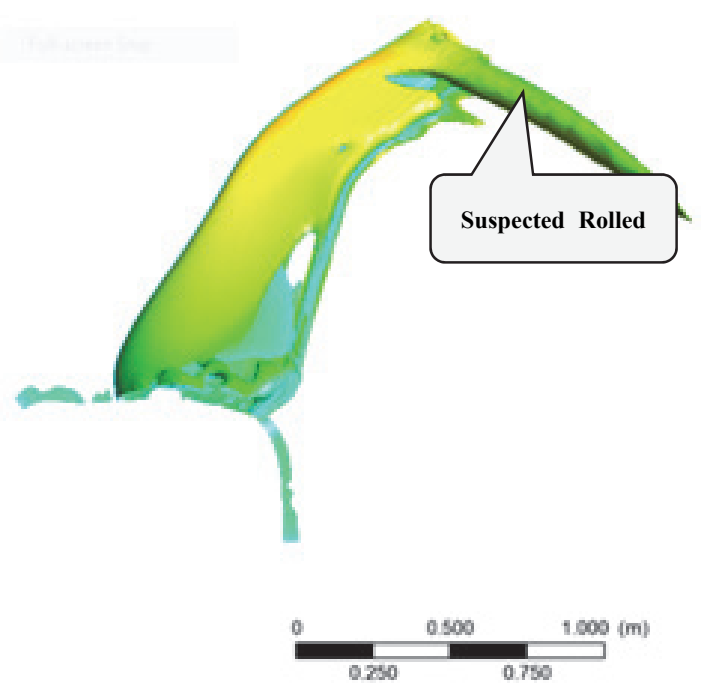

(b) Vortex core visualization at $12 \mathrm{~m} / \mathrm{s}, 137.5 \mathrm{RPM}$

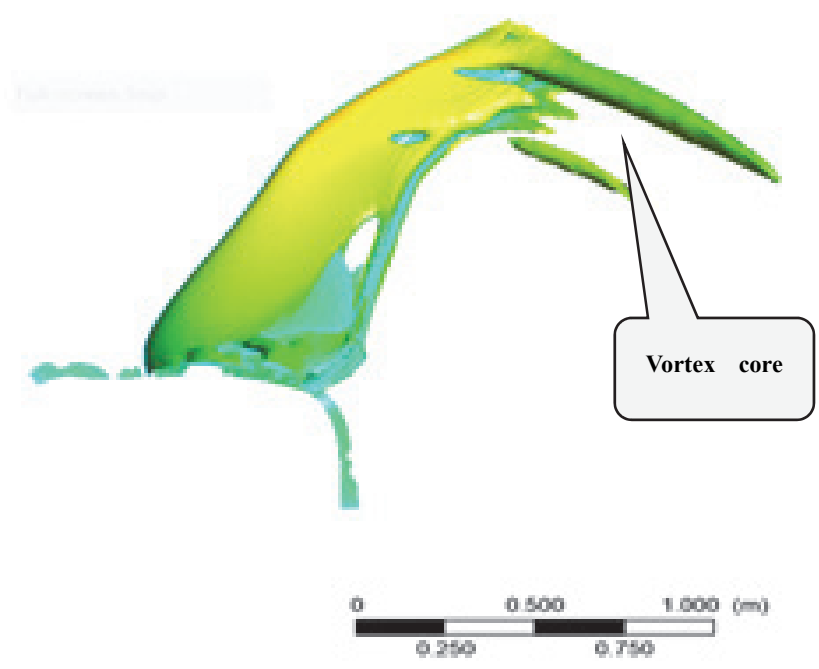

(c) Vortex core visualization at $12 \mathrm{~m} / \mathrm{s}, 136 \mathrm{RPM}$

Figure 6. Vortex-core visualization at speed 12 m/s 140 RPM, 137.5 RPM, 136 RPM

The laminar area shifts downward toward the hub. The decelerated region is lowered; the accelerated region is widened as its borderline is contracted. Then at the lower rotation that is at 120 RPM, the whole area has turned into a stall region. This is related to the visualization of the vortex cores, shown in Fig. 5. The data was taken at a speed of $12 \mathrm{~m} / \mathrm{s}$ in the transition region with variations of rotation 137.5, 136, 135, 120 and 90 RPM.

Vortex-core visualization at speed $12 \mathrm{~m} / \mathrm{s}$ 140, 137.5 and 136 RPM are displayed in Fig. 6 and at 135,120 and 90 RPM are displayed in Fig. 7, using the Q vortex identification criterion for each RPM (Chen et al., 2015). Plotted in Fig. 6 and 7 are iso-surfaces of instantaneous $Q=2.5 \times 105$ s- 2 colored by stream wise velocity. As illustrated in Fig. 6, at a speed of $12 \mathrm{~m} / \mathrm{s}$, a vortex core appears at 137.5, 136 and $135 \mathrm{RPM}$ and continues to stay visible in Fig. 7 for rotation 135, 120 and 90 RPM. In Fig. 6 and 7, at rotation 136 and 135 RPM, it indicates the presence of additional vortex cores so that the vortex core becomes 2 pieces.

At Fig. 8, the sketch of the measurement of the vortex center axial velocity and pressure along the trajectory of the core is illustrated by the result of the Q-criterion algorithm. Along the vortex core, the radially parallel planes are drawn to determine the center of the vortex, and then the axial velocity and pressure of the vortex center along the core could be calculated. Here, Ulocal $(\mathrm{z})$ is the virtual local velocity at the measured location (z). Fig. 9 illustrates the axial flow velocity ratio Uc/Ulocal (z). Fig. 10 shows the coefficient of pressure graphs of both vortices along the vortex cores for wind speeds of $12 \mathrm{~m} / \mathrm{s}$ at 180, 150, 145, 140, 136 and 135 RPM variations, 
where it is clearly seen here the coexistences of the 2 vortex centers. The velocity ratio Uc/Ulocal (z) along the vortex cores have been measured based on the vortex core trajectory, displayed in Fig. 8.
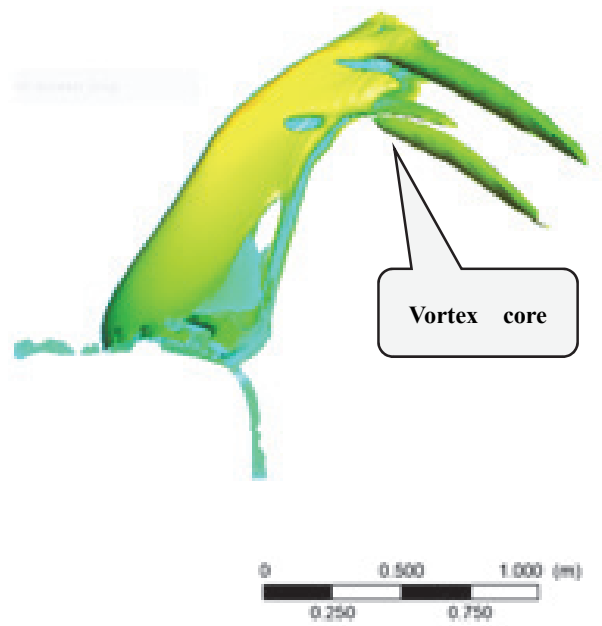
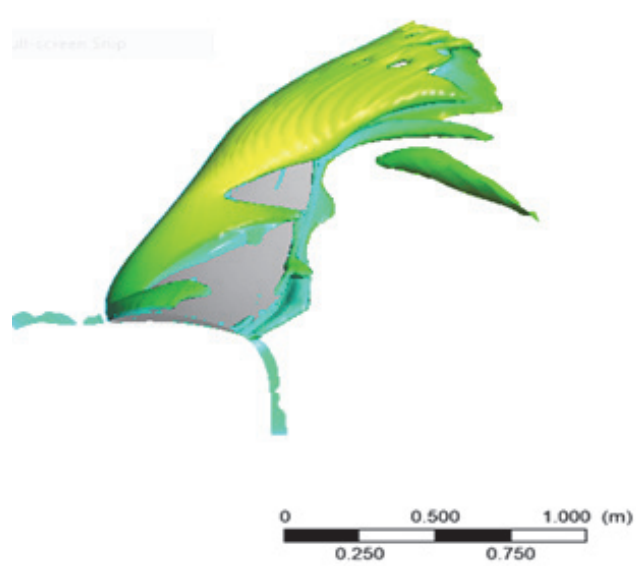

(b) Vortex core visualization at $12 \mathrm{~m} / \mathrm{s}, 120 \mathrm{RPM}$

(a) Vortex core visualization at $12 \mathrm{~m} / \mathrm{s}, 135 \mathrm{RPM}$

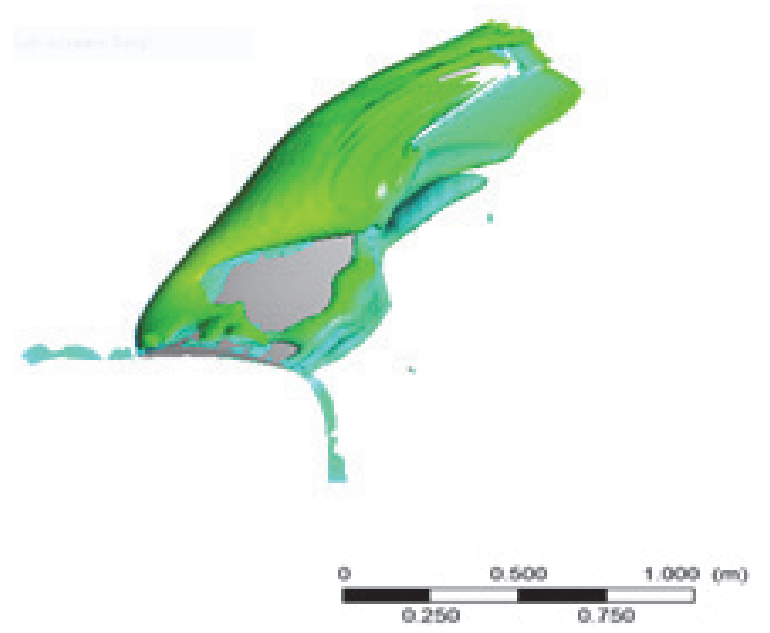

(c) Vortex core visualization at $12 \mathrm{~m} / \mathrm{s}, 90 \mathrm{RPM}$

Figure 7. Vortex-core visualization at speed 12 m/s 135 RPM, 120 RPM, 90 RPM

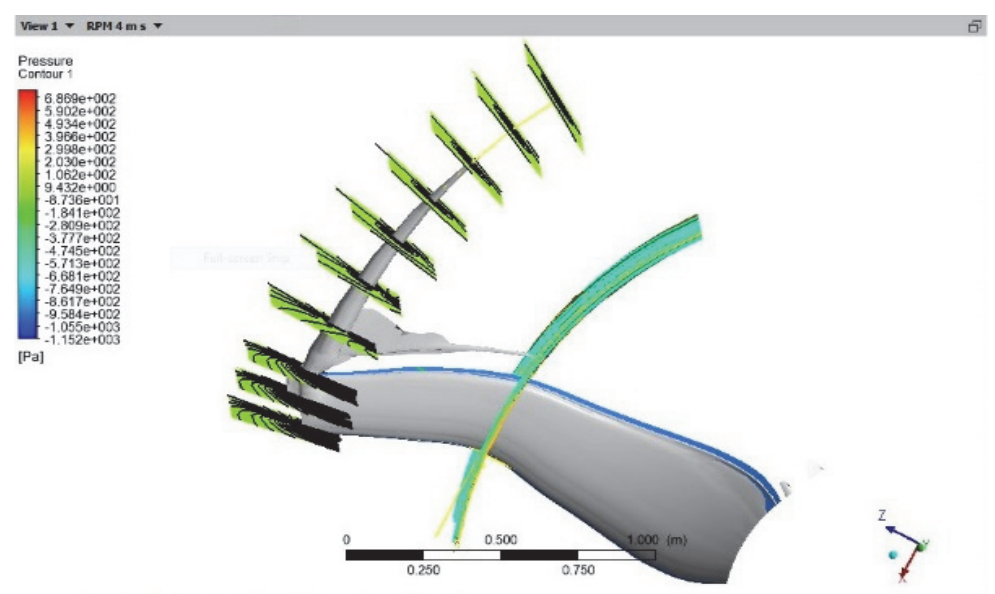

Figure 8 . The sketch of the measurement of the vortex center axial velocity and pressure along the core 
3.1.3 The strength of the vortex center

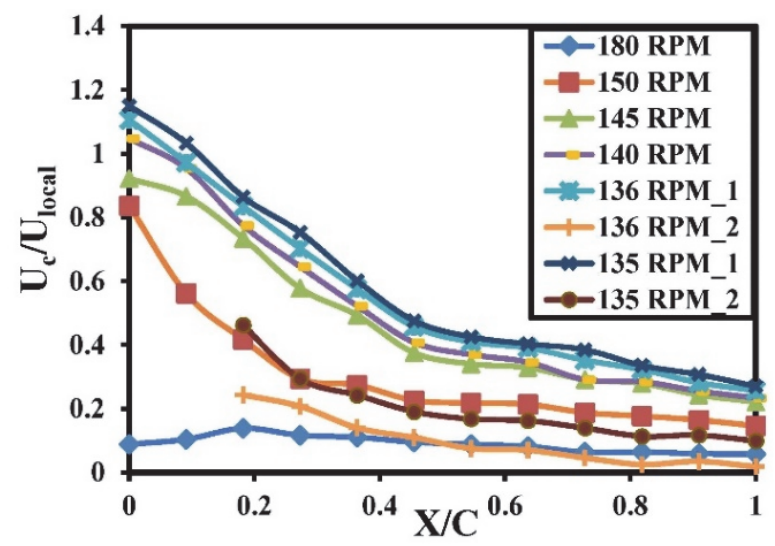

Figure 9. Graph of Uc/Ulocal (z)against X/C for various RPMs

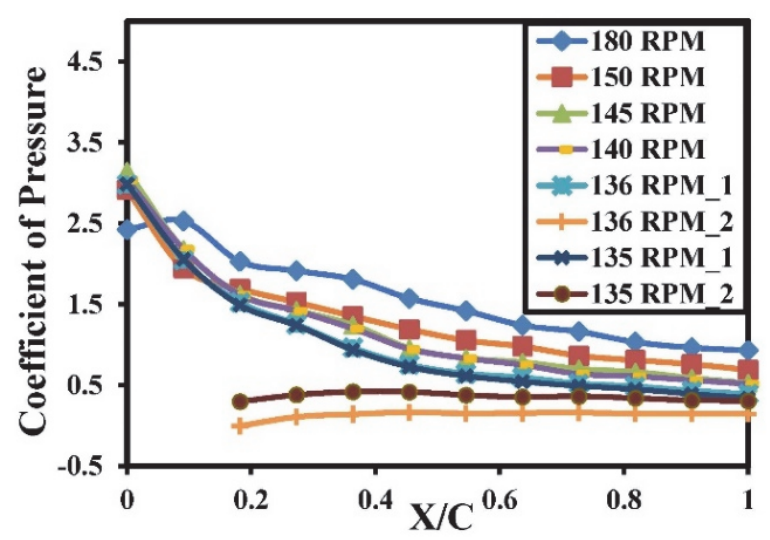

Figure 10. Graph of $\mathrm{Cp}$ against $\mathrm{X} / \mathrm{C}$ for various RPMs

\subsection{The Sharp-Edged Blade}

3.2.1The Limiting Streamline of the Sharp-Edged Wind Turbine Blade

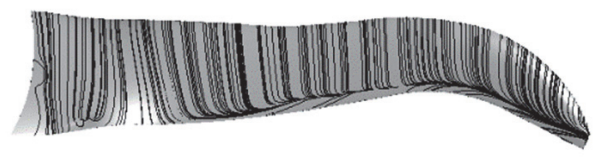

a) $12 \mathrm{~m} / \mathrm{s} 270 \mathrm{RPM}$ full laminar, start to become turbulent

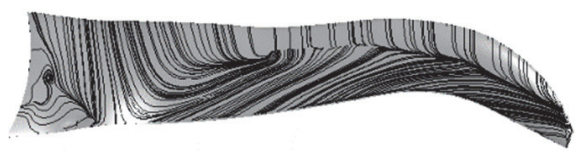

c) $12 \mathrm{~m} / \mathrm{s} 210$ RPM Surface $3 / 4$ turbulence

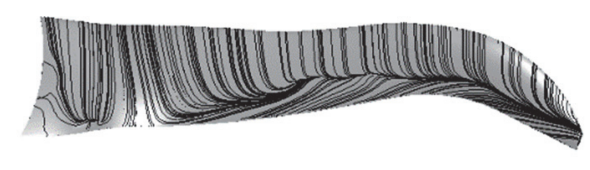

b) $12 \mathrm{~m} / \mathrm{s} 240$ RPM surface $1 / 4$ turbulence

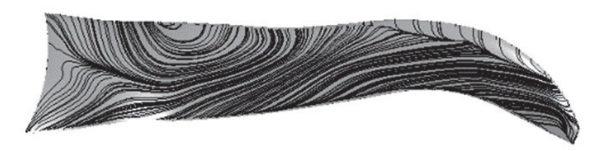

d) $12 \mathrm{~m} / \mathrm{s} 180$ RPM surface full turbulence

Figure 11. Limiting streamlines at the surface of the blade. The sample data was taken at a speed of $12 \mathrm{~m} / \mathrm{s}$ on the transition area with variation $137.5,136,135$ and $120 \mathrm{RPM}$ 
Figure 11 displays the limiting streamline on the blade surface at a wind speed of $12 \mathrm{~m} / \mathrm{s}$ for some variations of rotation 270, 240, 210 and 180 RPM. At a heavier load at 270 toward 180 RPM, the rotation decreases as the more area occupied by turbulence region, therefore the laminar region further shifts upward, more reduced toward the tip as shown in Fig. 11b, c and d. The laminar area shifts downward in the lower span, as the decelerated region of the disturbed region is lowered, the accelerated region is widened as its borderline decreases. Then at the lower rotation that is at 120 RPM, the whole area has turned into a stall region.

This is the particular case of the flow visualization of a backward wind turbine with a sharp pointed blade, where its vortex cores illustrated in Fig. 12. The data has been taken at the speed of $12 \mathrm{~m} / \mathrm{s}$ and at rotation 270, 180, 150 and 135 RPM. At Fig. 13, at rotation 187.5 and 180 RPM, it indicates the presence of additional vortex cores so that the vortex core becomes 2 pieces. Fig. 13b demonstrates the coefficient of pressure. Figure 13a shows the axial flow velocity ratio Uc/Ulocal (z) along the vortex cores at 270, 180, 150 and 135 RPM.

\subsubsection{The flow visualization of the vortex center}

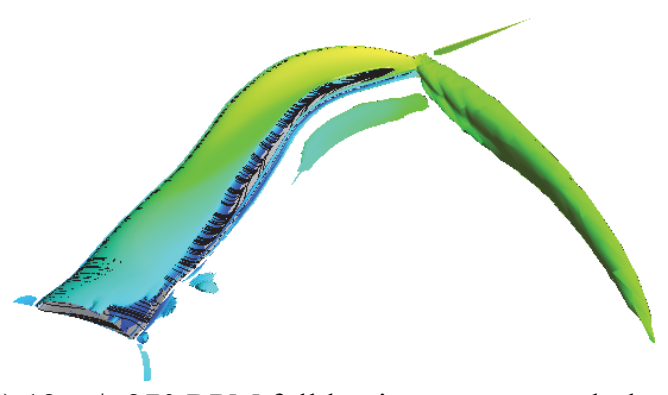

(a) $12 \mathrm{~m} / \mathrm{s} 270 \mathrm{RPM}$ full laminar, start to turbulent

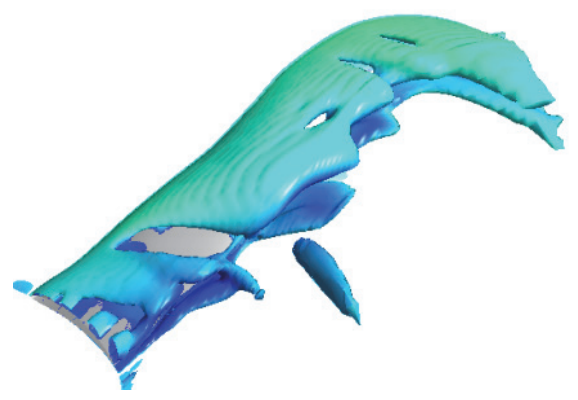

(c) $12 \mathrm{~m} / \mathrm{s} 150 \mathrm{RPM}$

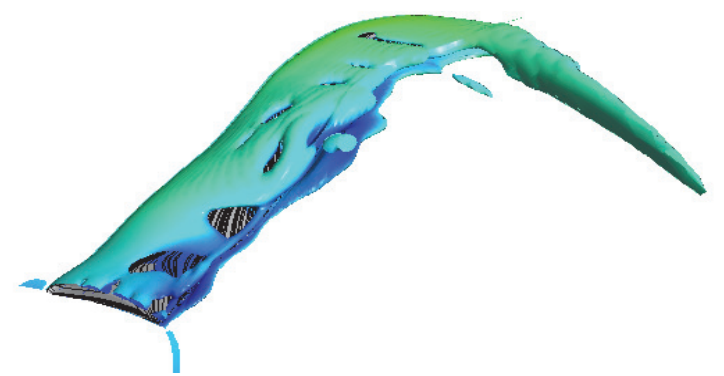

(b) $12 \mathrm{~m} / \mathrm{s} 180 \mathrm{RPM}$

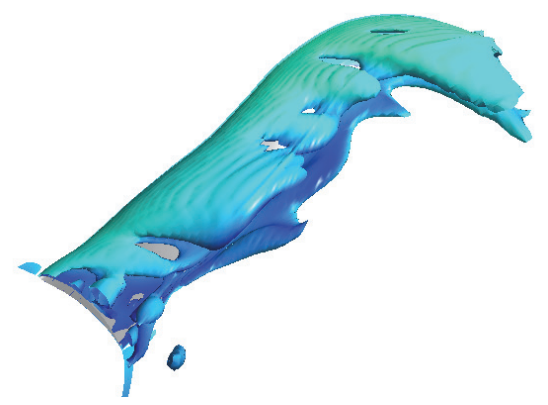

(d) $12 \mathrm{~m} / \mathrm{s} 135 \mathrm{RPM}$

Figure 12. Vortex-core flow visualization around forward wind turbine blade at the speed of $12 \mathrm{~m} / \mathrm{s}$ and at 270 , 180,150 and 135 RPM

\subsubsection{The Strength and Trajectory of the Vortex Center}

In order to show the effectiveness of the energy transform by the wind turbine, one could introduce the coefficient of turbine blade axial force, CAF and the coefficient of turbine blade circumferential force, CTF which have been normalized, divided by $1 / 2 \times \rho \times U 2$, where $\rho$ is density of air, and $U$ is the free stream velocity. Fig. 17 displays the coefficient of turbine blade axial force, CAF is high and the coefficient of turbine blade axial force, CTF is low, for 3-D backward wind turbine blade CFD model with a sharp-edged blade at 270 RPM at a lighter load. 


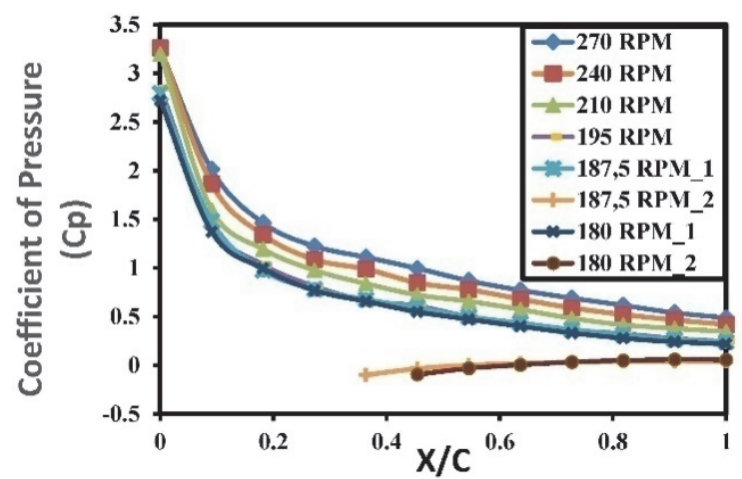

(a)

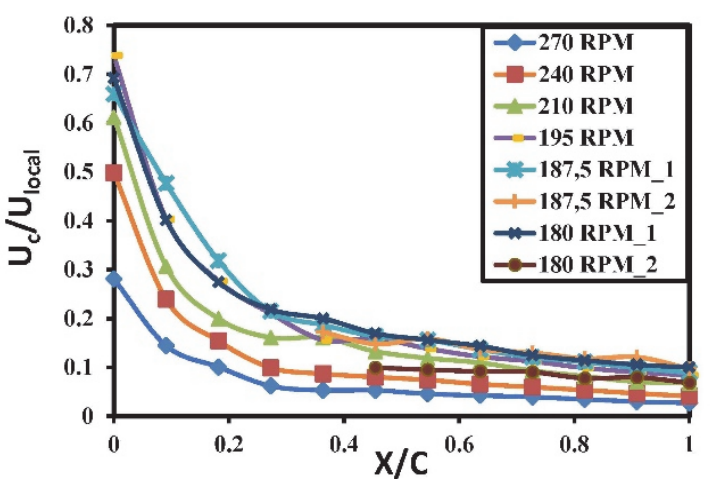

(b)

Figure 13. The plots of (a) the Uo/Ulocal (z) and (b) the coefficient of pressure to X/C for different RPM at 12 $\mathrm{m} / \mathrm{s}$

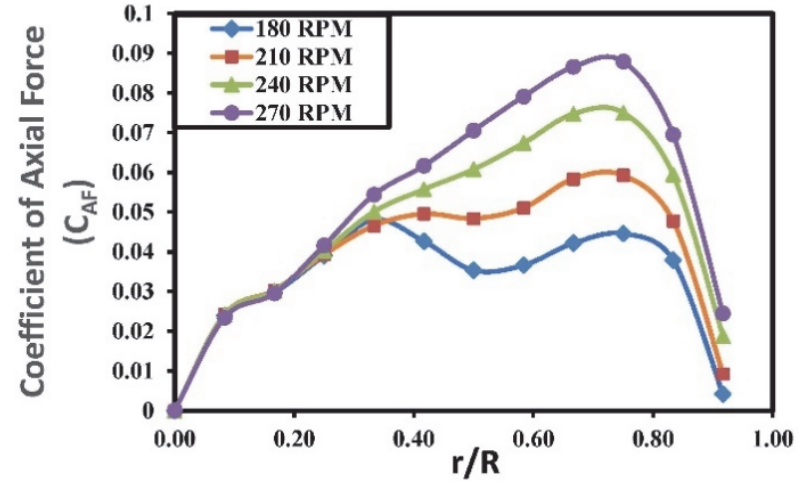

(a)

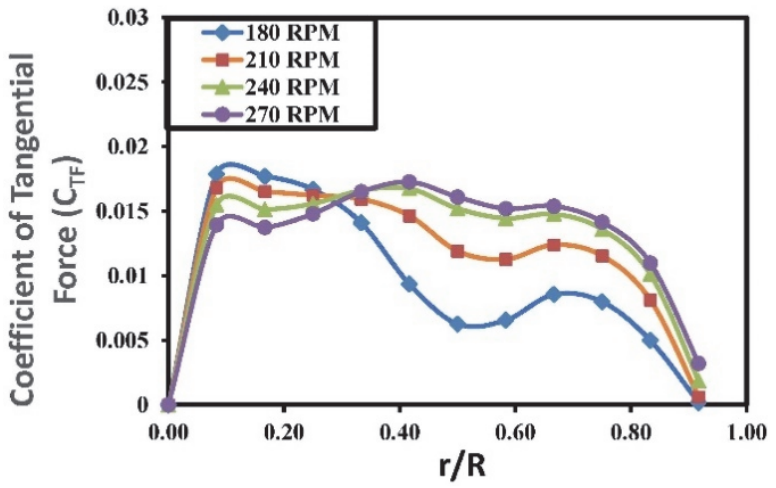

(b)

Figure 14. Graphs of coefficient of axial and tangential forces against the \% blade span at $12 \mathrm{~m} / \mathrm{s}$

\section{Discussion}

\subsection{The Method of Vortex Dynamics Analysis of 3D Wind Turbine Blades}

In turbulence region, rolled-up vortex effect could generate a vortex. It needs vortex dynamics analysis of 3-D flow pattern to show the interchange between rotational kinetic vortex energy and potential energy (negative pressure energy). First, the negative pressure energy on the surface could generate the lift on the surface, which could change into tangential energy again. Secondly, part of the kinetic rotational vortex energy could wipe out the turbulent area, so strong that could laminarize the turbulent area. Fortunately, the effect could only happen in wind turbine blades with a fin or with a helicopter head blade (Sutrisno et al., 2017).

It has been mentioned above that the significance of the rolled-up vortex effect, that is the quantified measurement of the vortex developed, the tip vortex and the vortex breakdown have not been evaluated, that one could not characterize more detailed about the strength of their vortices axial speed and negative pressure of the vortex centers including their surface pressure distribution to generate effective tangential powers. Unparalleled within aeronautical and aerospace engineering, the turbulent structures, topology, leading-edge vortex structure and second order turbulences in wind turbine blades sciences, especially for the 3-D wind turbine blades, other than straight blades, have not been investigated as much. The employment of the algorithm Q-criterion so far has not been combined with the footprint of the streamline for the vortex development, that incomplete picture of the vortex dynamics has not been concluded.

In order to have the whole picture of the vortex dynamics of the system, the authors would like to introduce the method to analyze vortex dynamics simulation. The method combines 2 parts, the flow visualization techniques and the analysis of the Q-criterion algorithm measurement result.

The first part, a) the flow visualization processes with several steps, first steps to plot the limiting streamline, drawing footprint from time to time or one variable to the next. Here, one could describe the changes from either 
laminar zone, turbulent zone, deceleration or acceleration zones and maybe the existence of rolled-up vortex effect. Next step, as the result of the Q-criterion algorithm, it will be shown the flow visualization plots of the main vortex center which may also generate the second vortex center. This vortex center can be a tip vortex if at the blade tip or could be a rolled-up vortex if in the middle of the blade. Rolled-up vortex, according to (Sutrisno et al., 2017), can cause stall delay or can cause vortex core and breakdown, can give laminarization impact on the turbulent area that has been occupied. This phenomenon is strongly obvious, for the case of finned wind turbine blade or the blade with helicopter head (Sutrisno et al., 2017).

The second part, b) the analysis to the quantified measurement of the Q-criterion algorithm due to the vortex, to produce i) the strength of the vortex center which is the axial velocity of the vortex center with respect to the local free stream velocity Uo/Ulocal(z) and the pressure coefficient $\mathrm{Cp}$ of the vortex center, along the vortex center or the measures of the pressure coefficient of the total pressure loss-center over the area under study, ii) the measurement of the trajectory height and spanwise location of the vortex center or the center of the total pressure loss. It means that if the tip vortex and the vortex breakdown have not been evaluated than one could not characterize more detailed about their surface pressure distributions to generate effective tangential powers

In this paper, the CFD model using detached-eddy simulation (DES) to simulate the characteristic of 3-D backward wind turbine blade model, the blunt and the sharp edge, have been examined. In this case one should show the complete illustration of their vortex dynamic phenomena, display the footprint of the streamline for the vortex development, the results of Q criterion algorithm employment to show vortex center, vortex core and breakdown location with respect to free stream velocity. Here, the results of measurement of the trajectory height and spanwise location of the vortex center are not important.

The purposes of the analysis are to comprehend the different between the blunt and the sharp edge wind turbine blades, step by step, the function of 3-D flow vortex mechanism patterns, to measure the strength of their vortices axial speed and negative pressure and trajectory of the vortex centers including their surface pressure distribution to generate effective tangential powers, the strength of the vortex center the length of the vortex core, the loss of energy with respect to the tangential local blade velocity.

\subsection{The Appearance of Rolled-Up Vortex Effect}

The purpose of the investigation was to measure the massive swirl of the rolled-up vortex effect, the strength of the vortex center, the length of the vortex core, the energy loss with respect to the tangential local blade velocity, the stream line distribution and pressure coefficient distribution

Fig. 5 to 9 illustrate the performance of the 3-D backward wind turbine blade CFD model with a blunt edge for $\mathrm{U}=12 \mathrm{~m} / \mathrm{s}$ at 180,150,145, 140, 136 and $135 \mathrm{RPM}$. The limiting streamlines have been reported by Sutrisno et al. The Fig. 5 demonstrates the limiting streamlines at the surface of the blade at 137.5, 136, 135 and 120 RPM, (Sutrisno, Deendarlianto, et al., n.d.). Fig. 6 and 7 display vortex-center flow visualization at speed $12 \mathrm{~m} / \mathrm{s} 140$, 137.5, 136 RPM and s 135, 120, 90 RPM. Fig. 9 denotes Uo/Ulocal (z) with respect to X/C at 12 m/s. Fig. 10 show $\mathrm{Cp}$ with respect to $\mathrm{X} / \mathrm{C}$ at $12 \mathrm{~m} / \mathrm{s}$.

Fig. 11 to 14 show the performance of the 3-D backward wind turbine blade CFD model with a sharp edge at a speed of $12 \mathrm{~m} / \mathrm{s}$ at 270, 240, 210 and 180 RPM. Fig. 11 illustrates the limiting streamlines at the surface of the blade. Fig. 12 displays the vortex-center flow visualization at speed $12 \mathrm{~m} / \mathrm{s}$ 270, 180, 150, 135 RPM. Fig. 13 presents $\mathrm{Cp}$ and Uo/Ulocal (z) with respect to X/C at $12 \mathrm{~m} / \mathrm{s}$ and Fig. 14 shows the axial force coefficient CAF shows the tangential force coefficient CTF along the span at $12 \mathrm{~m} / \mathrm{s}$.

Table 1. Characteristics comparison between 3-D backward wind turbine blade CFD model blunt edge for $\mathrm{U}=10$ $\mathrm{m} / \mathrm{s}$ at 180, 150, 145, 140, 136 and $135 \mathrm{RPM}$, with that of sharp-edged blades for $\mathrm{U}=10 \mathrm{~m} / \mathrm{s}$ at $270,180,150$ and 135 RPM. Axial force coefficient CAF for different for backward wind turbine blade with blunt edge derived from Sutrisno et al. (Sutrisno, Deendarlianto, et al., n.d.), derived from Fig. 5, and that with sharp-edged derived from Fig. 14. The Velocity ratio variation Uo/Ulocal (z) along the vortex core, showing the "Vortex breakdown location" derived from Fig. 10 and 16b and pressure coefficient variation $\mathrm{Cp}$ along the vortex core from Fig. 9 and $16 \mathrm{c}$, are functions of $\mathrm{xc} / \mathrm{crc}$. VBD location is the vortex breakdown location. 


\begin{tabular}{|c|c|c|c|c|c|c|c|}
\hline & $\begin{array}{l}\text { Wind Turbine } \\
\text { Blade (RPM) }\end{array}$ & $180 \mathrm{RPM}$ & 150 RPM & 145 RPM & $140 \mathrm{RPM}$ & $136 \mathrm{RPM}$ & $135 \mathrm{RPM}$ \\
\hline \multirow{5}{*}{$\begin{array}{l}\text { 3D Backward Wind } \\
\text { Turbine Blade } U=12 \\
\text { m/s (BLUNT BLADE) }\end{array}$} & $\mathrm{U}_{\mathrm{c}} / \mathrm{U}_{\text {local }}(\mathrm{z}) \max$ & 0.0 & 0.8 & 0.9 & 1.0 & 1.05 & 1.1 \\
\hline & VBD location & - & - & - & 0.0 & 0.1 & 0.2 \\
\hline & $\mathrm{C}_{\mathrm{AF}}$ (total) & 0.08 & 0.065 & 0.063 & 0.0607 & 0.0594 & 0.0621 \\
\hline & $\mathrm{C}_{\mathrm{TF}}$ (total) & 0.03 & 0.03 & 0.02542 & 0.02546 & 0.02563 & 0.02574 \\
\hline & $-\mathrm{C}_{\mathrm{p}} \max$ & 2.5 & 2.9 & 3.1 & 3.0 & 3.0 & 3.0 \\
\hline \multirow{6}{*}{$\begin{array}{l}\text { 3D Backward Wind } \\
\text { Turbine Blade U = } 12 \\
\text { m/s (SHARP EDGED } \\
\text { BLADE) }\end{array}$} & $\begin{array}{l}\text { Wind Turbine } \\
\text { Blade (RPM) }\end{array}$ & $270 \mathrm{RPM}$ & $240 \mathrm{RPM}$ & $210 \mathrm{RPM}$ & 195 RPM & 187 RPM & $180 \mathrm{RPM}$ \\
\hline & $\mathrm{U}_{\mathrm{c}} / \mathrm{U}_{\text {local }}(\mathrm{z}) \max$ & 0.65 & 0.73 & 0.6 & 0.75 & 0.7 & 0.7 \\
\hline & VBD location & - & - & - & - & - & - \\
\hline & $\mathrm{C}_{\mathrm{AF}}(\max )$ & 0.09 & 0.075 & 0.06 & 0.0506 & 0.0475 & 0.04 \\
\hline & $\mathrm{C}_{\mathrm{TF}}(\max )$ & 0.020 & 0.021 & 0.022 & 0.01754 & 0.017703 & 0.023 \\
\hline & $-C_{p} \max$ & 2.7 & 2.8 & 3.0 & 3.3 & 3.3 & 2.7 \\
\hline
\end{tabular}

Table 1 characterizes the comparison between 3-D backward wind turbine blade CFD model blunt edge with that of sharp-edged blades. Axial force coefficient CAF for different for backward wind turbine blades with blunt edge derived from Sutrisno et al. (Sutrisno, Deendarlianto, et al., n.d.), derived from Fig. 5, is compared with that employing sharp-edged derived from Fig. 14. The Velocity ratio variation Uo/Ulocal (z) along the vortex core, showing the "Vortex breakdown location" derived from Fig. 10 and $16 \mathrm{~b}$ and pressure coefficient variation $\mathrm{Cp}$ along the vortex core from Fig. 9 and 16c, are functions of xc/crc.

Furthermore, Table 1 illustrates that the coefficient of turbine blade axial force, CAF, for 3-D backward wind turbine blade CFD model with blunt edge blade is stronger compared with the sharp-edged blade. The velocity ratio variation Uo/Ulocal (z), or axial vortex center velocity with respect to free stream velocity along the vortex center, with blunt edge blade is much stronger compared with the sharp-edged blade. The "vortex breakdown location" for 3-D backward wind turbine blade CFD model with blunt edge blade, derived from Fig. 9, is farther than that with sharp-edged blade derived from Fig. 16b. And the pressure coefficient variation $\mathrm{Cp}(\max )$ along the vortex center for 3-D backward wind turbine blade CFD model with blunt edge blade, is stronger for 270 RPM to 180 RPM, while that with sharp-edged is stronger for 180 to 135 RPM.

The previous paper (Sutrisno, Rochmat, et al., n.d.) stated that the end of the laminar region of the backward wind turbine blades with blunt edge increasingly shrink. Next, a) As the laminar region of the backward wind turbine blades with blunt edge begins to shrink at $U=12 \mathrm{~m} / \mathrm{s}$, at $140 \mathrm{RPM}$, the second vortex center appears at the laminar end of the region, it is thought to be due to a type of vortex rolled-up effect occurring there. b) The main vortex or the tip vortex, of the blunt edge backward wind turbine blades, reaches Uo/Ulocal (z) $\geq 1.0$ with maximum negative pressure coefficient -Cpmax $\geq 3.0$. c) On the backward wind turbine blades sharp-edged blade tip, the vortex center is weak, that there vortex cores do not occur. The sharp-edged blade has a higher $\mathrm{CAF}$ value, with a lower CTF, it will rotate at a higher RPM.

\subsection{Laminar zone deflects the flow and creates rolled-up vortex effect}

The method to analyze vortex dynamics simulation can be elucidated by the appearance of rolled-up vortex effect on the backward wind turbine blades, for blunt blades generally have a strong vortex center, typically a vortex tip, which can be a vortex core, but also generates a second weak vortex center. This second vortex center appears due to the rolled-up vortex effect as a result of the swept effect in the mid-zone of the backward wind turbine blades.

The effect of rolled-up vortex on the backward wind turbine blades can be explained for a sharp pointed blade generally has a weak vortex center, generally a tip vortex, which is difficult not a vortex core, but may generate a second weak vortex center, which is quite difficult to be identified. It is also expected that this second vortex center appears due to the vortex rolled-up effect due to the swept effect in the mid-zone of the wind turbine blades backward. The appearance of the rolled-up vortex would be explained below.

From flow visualization, the limiting streamline, it can be seen that for both blades (blunted and pointed blades) the appearance of rolled-up vortex is caused by what happens in the laminar zone, because: laminar zone acts like the base of a hill or a mountain, it will deflect the direction of any flow rushing toward it, it will create rolled-up vortex, rolled-up vortex effect. 
On the backward wind turbine blades with blunt blades, with the blade bending towards the blade base, the turbulent area arises from the blade tip, namely the strongly accelerated disturbed region, so after some time the laminar region becomes eroded backward, toward the base of the blade. The laminar border zone retreats, giving rise to a rolled-up vortex that evokes a weak vortex center, but it is still clearly visible.

On the backward wind turbine blades with sharp pointed tip, with the bending of the blade towards the blade tip, the narrowly pointed blade tip cause weakening the vortex tip, the gnawing of the laminar region is very weak at the tip. Trudging in the middle is stronger, so the laminar area begins to disappear, and the gnawing runs toward the tip of the blade. Open the possibility the appearance of the rolled-up vortex in the middle of the blade, so come out a weak second vortex center.

\section{Conclusion}

In this paper the method to analyze vortex dynamics simulation was introduced, consisted of flow visualization part and detailed measurement part. a) The flow visualization part utilizes the limiting (the surface of) streamline and uses Q-criterion to demonstrate the vortex appearance, which is to describe the pattern of vortex tip, which is generally the main vortex center which can be followed by second vortex center and rolled-up vortices pattern. b) The detailed measurement part, the quantified measurement of the Q-criterion algorithm, which could illustrate the vortex strength (axial part of the vortex velocity and pressure coefficient of the vortex) along the vortex center of the vortex center. With this method one can explain the function of 3-D flow vortex mechanism patterns on 3-D backward wind turbine blade, the interchange between kinetic and potential energies, the utilization of very strong vortex which could lose energy, generate lift, produce tangential mechanical power. In 3-D wind turbine blades at high-speed heavy loads or low RPM, most likely rolled-up vortices occur. The bigger the rolled-up vortex, the lower the wind turbine blades 's efficiency.

The method to analyze vortex dynamics simulation can be elucidated by the appearance of rolled-up vortex effect on the backward wind turbine blades, for blunt blades generally have a strong vortex center, typically a vortex tip, which can be a vortex core, but also generate a second weak vortex center. This second vortex center appears due to the vortex rolled-up effect, the swept effect in the mid-zone of the backward wind turbine blades.

The effect of the rolled-up vortex on the backward wind turbine blades can be explained for a sharp pointed blade generally has a weak vortex center, generally a tip vortex, which is difficult / not to be a vortex core, but may generate a second weak vortex center, which is quite difficult to recognize. It is also expected that this second vortex center appears due to the vortex rolled-up effect due to the swept effect in the mid-zone of the wind turbine blades backward. From flow visualization, the limiting streamline, it can be seen that for both blades (blunted and pointed blades) the appearance of rolled-up vortex is caused by what happens in the laminar zone, because: laminar zone acts like the base of a hill or a mountain, it will deflect the direction of any flow rushing toward it, it will create rolled-up vortex, rolled-up vortex effect.

On the backward wind turbine blades with blunt blades, with the blade bending towards the blade base, the turbulent area arises from the blade tip, namely the strongly accelerated disturbed region, so after some time the laminar region becomes eroded backward, toward the base of the blade. The laminar border zone retreats, giving rise to a rolled-up vortex that evokes a weak vortex center, but it is still clearly visible. On the backward wind turbine blades with sharp pointed tip, with the bending of the blade towards the blade tip, the narrow pointed blade tip cause weakening the vortex tip, the gnawing of the laminar region is very weak at the tip. Trudging in the middle is stronger, so the laminar area begins to disappear, and the gnawing runs toward the tip of the blade. Open the possibility the appearance of the rolled-up vortex in the middle of the blade, so come out a weak second vortex center.

\section{Nomenclature}

$\mathrm{C}_{\mathrm{p}}=$ coefficient of pressure

$\mathrm{C}_{\mathrm{AF}}=$ the coefficient of turbine blade axial force, normalized by $1 / 2 * \rho^{*} \mathrm{U}^{2}$

$\mathrm{C}_{\mathrm{TF}}=$ the coefficient of blade circumferential force, normalized by $1 / 2 * \rho * \mathrm{U}^{2}$

$\mathrm{U}_{\mathrm{c}}=$ axial vortex core/center velocity

$\mathrm{U}_{\text {local }}(\mathrm{z})=$ virtual local velocity at $\mathrm{z}$

$\mathrm{U}_{\mathrm{c}} / \mathrm{U}_{\text {local }}(\mathrm{z}) \quad=$ axial vortex center velocity ratio

$\left.\mathrm{p}_{\mathrm{w}}(\mathrm{y})\right|_{\mathrm{z}} \quad=$ wall pressure at $\mathrm{z}$, along the $\mathrm{y}$-direction

$\mathrm{RPM}=$ the frequency of the rotor blade (rotation/minutes) 
$\mathrm{U}=$ free stream velocity $(\mathrm{m} / \mathrm{s})$

$\Psi \quad=$ swept angle

$\mathrm{y}+=$ a non-dimensional wall distance

$\mathrm{x}, \mathrm{y}=$ abscissa and ordinate

$\rho=$ density of air

$\mathrm{U} \quad=$ free stream velocity

\section{Acknowledgement}

This work has been carried out with a support of the Department of Higher Education, the Republic of Indonesia 143/LPPM/2015. The authors would like to express sincere gratitude to Dr. Prajitno, MT for time spent in serious discussion, helpful suggestion and useful conceptual contribution. We would like to thank also our students Firdaus, Wega, Zain, Yogi, my laboratory staffs, Waji and Min, their help in construction work and conducting data management are gratefully acknowledged.

\section{References}

Brocklehurst, A., \& Barakos, G. N. (2013). A Review of Helicopter Rotor Blade Tip Shapes. Progress in Aerospace Sciences, 56, 35-74. https://doi.org/10.1016/j.paerosci.2012.06.003

Cai, J., Tsai, H. M., Luo, S., \& Liu, F. (2011). Design of an Optimal Wing-Body Configuration to Delay Onset of Vortex Asymmetry. AIAA Journal, 49(1), 164-171. https://doi.org/10.2514/1.J050595

Calderon, D.E, Wang, Z, \& Gursul, I. (2012). Three-dimensional measurements of vortex breakdown. Experiments in Fluids, 53(May), 293-299. https://doi.org/10.1007/s00348-012-1317-1

Chen, M., Liu, P., Guo, H., \& Qu, Q. (2015). Effect of sideslip on high-angle-of-attack vortex flow over close-coupled canard configuration. Journal of Aircraft, 53(1), 217-230. https://doi.org/10.2514/1.C033305

Cummings, R. M., \& Schutte, A. (2010). An Integrated Computational/Experimental Approach to UCAV Stability \& Control Estimation: Overview of NATO RTO AVT-161. In AIAA Applied Aerodynamics Conference (pp. 1-23). https://doi.org/10.2514/6.2010-4392

Erm, L. P., \& Ol, M. V. (2012). An Assessment of the Usefulness of Water Tunnels for Aerodynamic Investigations, (August 2010).

Ghoreyshi, M., Cummings, R. M., Ronch, A. Da, \& Badcock, K. J. (2013). Transonic Aerodynamic Load Modeling of X-31 Aircraft Pitching Motions. AIAA Journal, 51(10), 2447-2464. https://doi.org/10.2514/1.J052309

Ghoreyshi, M., Korkis-kanaan, R., Jirásek, A., Cummings, R. M., \& Lofthouse, J. (2016). Simulation validation of static and forced motion flow physics of a canard configured TransCruiser Aerospace Science and Technology, 48, 158-177. https://doi.org/10.1016/j.ast.2015.11.008

Gursul, I. Ã., Wang, Z., \& Vardaki, E. (2007). Review of flow control mechanisms of leading-edge vortices. Progress in Aerospace Sciences, 43, 246-270. https://doi.org/10.1016/j.paerosci.2007.08.001

Haller, G. (2005). An objective definition of a vortex. J. Fluid Mech, 525, 1-26. https://doi.org/10.1017/S0022112004002526

Han, C., \& Kinnas, S. A. (2013). Study on the Wake Shape behind a Wing in Ground Effect Using an Unsteady Discrete Vortex Panel Method. Open Journal of Fluid Dynamics, 3(December), 261-265. https://doi.org/10.4236/ojfd.2013.34032

Hirahara, H., Hossain, M. Z., \& Kawahashi, M. (2005). Testing basic performance of a very small wind turbine designed for multi-purposes, 30, 1279-1297. https://doi.org/10.1016/j.renene.2004.10.009

Ibrahim, I., \& New, T. (2015). Flow Separation Control of Marine Propeller Blades through Tubercle Modifications. In 10th Pacific Symposium on Flow Visualization and Image Processing.

Im, H. S., \& Zha, G. C. (2011). Delayed Detached Eddy Simulation of a Stall Flow Over NACA0012 Airfoil Using High Order Schemes. Proceedings of the 49th AIAA Aerospace Sciences Meeting Including the New Horizons Forum and Aerospace Exhibition, (January), 1-16.

Jee, S., \& Shariff, K. (2014). Detached-eddy simulation based on the v2-f model. In 7 Int Copnf ICCFD (Vol. 46, pp. 84-101). Hawai. https://doi.org/10.1016/j.ijheatfluidflow.2013.12.006 
Jin-Jun, W., \& Wang, Z. (2008). Experimental investigations on leading-edge vortex structures for flow over non-slender delta wings. Chinese Physics Letters, 25(7), 2550-2553. https://doi.org/10.1088/0256-307X/25/7/060

Kirkil, G., Constantinescu, G., \& Ettema, R. (2009). Detached Eddy Simulation Investigation of Turbulence at a Circular Pier with Scour Hole. Journal of Hydraulic Engineering, 135(11), 888-901. https://doi.org/10.1061/(ASCE)HY.1943-7900.0000101

Kwak, D., Nelson, R. C., Dame, N., \& Dame, N. (2010). Vortical Flow Control over Delta Wings with Different Sweep Back Angles Using DBD Plasma Actuators. In 5th Flow Control Conference (pp. 1-10). Chicago. https://doi.org/doi:10.2514/6.2010-4837

Larsson, J., \& Wang, Q. (2014). The prospect of using large eddy and detached eddy simulations in engineering design, and the research required to get there. Philosophical Transactions of the Royal Society A: Mathematical. Physical and Engineering Sciences, 372(2022), 1-15. https://doi.org/10.1098/rsta.2013.0329

Lee, J., Lee, Y., \& Lim, H. (2017). Effect of twist angle on the performance of Savonius wind turbine Effect of twist angle on the performance of Savonius wind turbine. Renewable Energy, 89(April 2016), 231-244. https://doi.org/10.1016/j.renene.2015.12.012

Lee, T., \& Su, Y. Y. (2012). Wingtip vortex control via the use of a reverse half-delta wing. Experiments in Fluids, 52(6), 1593-1609. https://doi.org/10.1007/s00348-012-1274-8

Liu, C., Liu, C., \& Ma, W. (2015). Rans, detached Eddy simulation and large Eddy simulation of internal Torque converters flows: A comparative study. Engineering Applications of Computational Fluid Mechanics, 9(1), 114-125. https://doi.org/10.1080/19942060.2015.1004814

Miller, G. D., \& Williamson, C. H. K. (1997). Turbulent Structures in the Trailing Vortex Wake of a Delta Wing. Experimental Thermal and Fluid Science, 14(96), 2-8.

Mitchell, A. M., \& Delery, J. (2001). Research into vortex breakdown control. Progress in Aerospace Sciences, 37(4), 385-418. https://doi.org/10.1016/S0376-0421(01)00010-0

Mockett, C. (2008). A comprehensive study of detached-eddy simulation. PhD Thesis: TU Berlin.

Morton, S. a, Kholodar, D., Billingsley, T., Forsythe, J. R., Wurtzler, K. E., Squires, K. D., ... Spalart, P. R. (2004). Multidisciplinary applications of detached-eddy simulation to separated flows at high Reynolds numbers (challenge 92). In Users Group Conference, 2004. Proceedings (pp. 103-111). https://doi.org/10.1109/DOD_UGC.2004.28

Muscari, R., Di Mascio, A., \& Verzicco, R. (2013). Modeling of vortex dynamics in the wake of a marine propeller. Computers and Fluids, 73, 65-79. https://doi.org/10.1016/j.compfluid.2012.12.003

Muscari, R., \& Mascio, A. Di. (2013). Detached Eddy Simulation of the flow behind an isolated propeller, (May), $218-226$.

Nelson, R. C., \& Pelletier, A. (2003). The unsteady aerodynamics of slender wings and aircraft undergoing large amplitude maneuvers. Progress in Aerospace Sciences, 39(2-3), 185-248. https://doi.org/10.1016/S0376-0421(02)00088-X

Nicolleau, F. C. G. A., Cambon, C., Redondo, J. M., Vassilicos, J. C., Reeks, M., \& Nowakowski, A. F. (2012). New approaches in modeling multiphase flows and dispersion in turbulence, fractal methods and synthetic turbulence. ERCOFTAC Series, 18, 9-22. https://doi.org/10.1007/978-94-007-2506-5

Sahin, B., Yayla, S., Canpolat, C., \& Akilli, H. (2012). Flow structure over the yawed nonslender diamond wing. Aerospace Science and Technology, 23(1), 108-119. https://doi.org/10.1016/j.ast.2011.06.008

Samimi, S., Davari, A. R., \& Soltani, A. M. R. (2013). Canard-Wing Interactions in Subsonic Flow *. Transactions of Mechanical Engineering, 37(M2), 133-147.

Sherry, M., Nemes, A., Lo Jacono, D., Blackburn, H. M., \& Sheridan, J. (2013). The interaction of helical tip and root vortices in a wind turbine wake. Physics of Fluids, 25(11). https://doi.org/10.1063/1.4824734

Sherry, M., Sheridan, J., \& Jacono, D. Lo. (2013). Characterisation of a horizontal axis wind turbine's tip and root vortices. Experiments in Fluids, 54(3). https://doi.org/10.1007/s00348-012-1417-y

Spalart, P. R. (2009). Detached-Eddy Simulation. Annual Review of Fluid Mechanics, 41(1), 181-202. https://doi.org/10.1146/annurev.fluid.010908.165130

Squires, K. D. (2004). Detached-eddy simulation: Current status and perspectives. In F. R., G. B.J., \& Métais O. 
(Eds.), Direct and Large-Eddy Simulation V. ERCOFTAC Series, vol 9. Springer, Dordrecht. https://doi.org/https://doi.org/10.1007/978-1-4020-2313-2_49

Sun, D., Li, Q., \& Zhang, H. (2013). Detached-eddy simulations on massively separated flows over a 76/40 double-delta wing. Aerospace Science and Technology, 30(1), 33-45. https://doi.org/10.1016/j.ast.2013.07.001

Sutrisno, Deendarlianto, Indarto, Iswahyudi, S., Bramantyo, M, A., \& Setyawan, B. W. (2017). Performances and Stall Delays of Three Dimensional Wind Turbine Blade Plate-Models with Helicopter-Like Propeller Blade Tips. Modern Applied Science, 11(10), 189. https://doi.org/10.5539/mas.v11n10p189

Sutrisno, Deendarlianto, Rochmat, T. A., Indarto, B. S. S., Iswahyudi, S., ... Djatmiko. (n.d.). The Structured and Unstructured CFD Characteristic Studies of 3-D Backward Wind Turbine Blades. In to be published.

Sutrisno, Prajitno, Purnomo, \& B.W. Setyawan. (2016). The performance \& flow visualization studies of three dimensional (3-D) wind turbine blade models. Modern Applied Science, 10(5), 132-144. https://doi.org/10.5539/mas.v10n5p132

Sutrisno, Rochmat, T. A., Setyawan, B. S., Iswahyudi, S., Wiratama, C., \& Widia K. (n.d.). CFD Studies on the Performance and Vortex Breakdown Flow Visualization of Chengdu J-10. To Be Published.

Travin, A., Shur, M., Strelets, M., \& Spalart, P. (1999). Detached-Eddy Simulations Past a Circular Cylinder. Flow Turbulence and Combustion, 63, 293-313. https://doi.org/10.1023/A:1009901401183

Zhang, R., \& Wu, J. (2012). Aerodynamic characteristics of wind turbine blades with a sinusoidal leading edge. Wind Energy, 15(April 2012), 407-424. https://doi.org/10.1002/we.479

\section{Copyrights}

Copyright for this article is retained by the author(s), with first publication rights granted to the journal.

This is an open-access article distributed under the terms and conditions of the Creative Commons Attribution license (http://creativecommons.org/licenses/by/4.0/). 